\title{
Soliton ratchetlike dynamics by ac forces with harmonic mixing
}

\section{Salerno, Mario; Zolotaryuk, Yaroslav}

\section{Published in:}

Physical Review E. Statistical, Nonlinear, and Soft Matter Physics

Link to article, DOI:

10.1103/PhysRevE.65.056603

Publication date:

2002

Document Version

Publisher's PDF, also known as Version of record

Link back to DTU Orbit

Citation (APA):

Salerno, M., \& Zolotaryuk, Y. (2002). Soliton ratchetlike dynamics by ac forces with harmonic mixing. Physical Review E. Statistical, Nonlinear, and Soft Matter Physics, 65(5), 056603.

https://doi.org/10.1103/PhysRevE.65.056603

\section{General rights}

Copyright and moral rights for the publications made accessible in the public portal are retained by the authors and/or other copyright owners and it is a condition of accessing publications that users recognise and abide by the legal requirements associated with these rights.

- Users may download and print one copy of any publication from the public portal for the purpose of private study or research.

- You may not further distribute the material or use it for any profit-making activity or commercial gain

- You may freely distribute the URL identifying the publication in the public portal

If you believe that this document breaches copyright please contact us providing details, and we will remove access to the work immediately and investigate your claim. 


\title{
Soliton ratchetlike dynamics by ac forces with harmonic mixing
}

\author{
Mario Salerno ${ }^{1}$ and Yaroslav Zolotaryuk ${ }^{2}$ \\ ${ }^{1}$ Dipartamento di Fisica “E. R. Caianiello” and Istituto Nazionale di Fisica della Materia (INFM), Universitá di Salerno, \\ I-84081 Baronissi, Salerno, Italy \\ ${ }^{2}$ Section of Mathematical Physics, IMM, Technical University of Denmark, DK-2800, Kgs. Lyngby, Denmark
}

(Received 16 October 2001; published 29 April 2002)

\begin{abstract}
The possibility of unidirectional motion of a kink (topological soliton) of a dissipative sine-Gordon equation in the presence of ac forces with harmonic mixing (at least biharmonic) and of zero mean, is presented. The dependence of the kink mean velocity on system parameters is investigated numerically and the results are compared with a perturbation analysis based on a point-particle representation of the soliton. We find that first order perturbative calculations lead to incomplete descriptions, due to the important role played by the solitonphonon interaction in establishing the phenomenon. The role played by the temporal symmetry of the system in establishing soliton dc motions that resemble usual soliton ratchets, is also emphasized. In particular, we show the existence of an asymmetric internal mode on the kink profile that couples to the kink translational mode through the damping in the system. Effective soliton transport is achieved when the internal mode and the external force get phase locked. We find that for kinks driven by biharmonic drivers consisting of the superposition of a fundamental driver with its first odd harmonic, the transport arises only due to this internal mode mechanism, while for biharmonic drivers with even harmonic superposition, also a point-particle contribution to the drift velocity is present. The phenomenon is robust enough to survive the presence of thermal noise in the system and can lead to several interesting physical applications.
\end{abstract}

DOI: 10.1103/PhysRevE.65.056603

PACS number(s): 05.45.Yv, 05.60.Cd, 05.45.Ac

\section{INTRODUCTION}

Transport phenomena based on nonlinear effects are at the heart of many problems in physics [1]. In this context it was generally believed that an ac force of zero mean cannot lead to directed nonzero currents. Recent studies of the so called ratchet effect have shown that this belief was wrong [2]. A ratchet system can be described as a Brownian particle in an asymmetric periodic potential, moving in a specific direction in presence of damping, under the action of ac forces of zero average. The origin of a net motion is associated to the breaking of the space-temporal symmetries of the system $[3,4]$, leading to the desymmetrization of Lévy flights (for Hamiltonian systems) [5], and to phase locking phenomena between the particle motion and the external driving force $[6,7]$.

This effect has a number of applications in various branches of physics and biology and is believed to be the basic mechanism for the functioning of biological motors (see reviews [2], and references therein). The ratchet effect, originally studied for Brownian particles, was generalized to dynamical systems [8] and to partial differential equations (PDE) of soliton-type, mainly in the overdamped regime [9], or with asymmetric potentials [10-14]. In the overdamped case, the damping in the system suppress all the degrees of freedom associated with the background radiation so that soliton ratchets become very similar to point-particle ratchets. For underdamped or moderately damped systems, however, the situation is quite different since the radiation field can interact with the soliton and influence the transport. Underdamped soliton ratchets in asymmetric potentials and driven by sinusoidal forces, were recently investigated in Refs. [13,14]. In particular, in Ref. [13] the basic mechanism underlying the phenomenon was identified in the existence of an asymmetric internal mode which couples, through the damping in the system, to the soliton translational mode. Effective transport was found when the internal mode and the external force were phase locked. Moreover, it was shown that the effect of soliton transport decreases with increase of the damping, the maximal transport being achieved in the underdamped regime.

On the other hand, it is known that for ordinary differential equation (ODE) systems, unidirectional transport is possible also in symmetric potentials if suitable asymmetric forces of zero average are applied. We remark that, although the term ratchet is usually used in connection with asymmetric potentials, one can adopt a more general definition by viewing the ratchet as the result of the breakage of the spatiotemporal symmetry of the system that relates orbits with opposite velocities (thus producing net motion independent on initial conditions). This can be achieved either by deforming the potential and using zero average symmetric forces, or by applying proper asymmetric forces with zero averages to systems with symmetric potentials. In the present paper we shall adopt this more general (symmetry based) definition of the ratchet phenomenon [15].

Since in concrete applications it is more easy to act on the temporal part (by using external forces) than on the spatial part (by inducing potential distortions) of a system, it is interesting to explore transport phenomena induced by asymmetric forces also in the case of soliton systems.

The present paper is just devoted to this problem. More precisely, we show that topological solitons of nonlinear PDEs with symmetric potentials can acquire finite drift velocities in the presence of biharmonic forces of zero average consisting of the superposition of two harmonics, the fundamental and one of its overtones (harmonic mixing drivers). Biharmonic forces were used in the literature to suppress 
chaos in dynamical systems and in soliton equations [16], as well as, to control the transport properties of single particle ratchets [7]. In the present paper we demonstrate, on the particular example of the sine-Gordon system, that biharmonic driving forces with certain symmetries can be effective to create soliton ratchets. The role played by the symmetry of the force in establishing the phenomenon is discussed. In contrast with previous studies, we find that the direction of the net soliton motion is totally controlled by the symmetry of the force and is independent from initial conditions.

The phenomenon is investigated both numerically, by direct simulations, and analytically, using soliton perturbation theory. We show that a first order perturbation analysis of the soliton dynamics captures only the qualitative features of the phenomenon, leading to poor quantitative agreements with numerical results. The reason of this discrepancy is ascribed to the soliton-phonon interaction that is obviously missing in a point-particle description (it arises only at the second order in the perturbation expansion [17]).

In general the situation can be described as follows. Besides a point-particle contribution to the drift velocity there is an equally important contribution coming from the solitonphonon interaction. This last manifests itself with the appearance of an internal oscillation on the soliton profile, asymmetric in space, which induces a net motion in a similar manner as described in Ref. [13]. In particular, we show that this oscillation can be phase locked to the external driving force and can couple to the kink translational mode, through the damping in the system. The energy, "pumped," by the ac field into the internal mode is then converted by the above mechanism into net motion of the kink. Internal oscillations on antikink profiles have opposite asymmetry compared to kinks, so that kink and antikink ratchets give rise to motion in opposite directions.

The dependence of the phenomenon on system parameters such as the damping in the system, frequency, amplitudes, relative phase of the biharmonic driving force, as well as, on the presence of white noise in the system, is investigated by means of direct numerical integrations of the sineGordon equation. The interaction of soliton ratchets with the boundaries of a finite system is also investigated. For reflecting edges we find that, depending on the initial velocity and position of the kink, the ratchet dynamics can be either destroyed or reflected as an antikink ratchet moving in the opposite direction. These results could be important for applications to physical systems such as long Josephson junctions, as we briefly discuss at the end of the paper.

The paper is organized as follows. In Sec. II we investigate the dynamics of the perturbed sine-Gordon equation, as a model for soliton ratchets in the presence of asymmetric forcing and damping, both in terms of symmetry arguments and first order perturbation theory. In Sec. III we study soliton ratchets by direct numerical integrations of the perturbed sine-Gordon system and provide a consistent interpretation of the phenomenon. Qualitative and quantitative features of soliton ratchets are compared with the predictions of the first order perturbation analysis. The phenomenon is investigated in the deterministic case (i.e., in absence of noise) and in the presence of a white noise term in the system. We find that the soliton ratchets are robust enough to overcome the presence of small amplitude noises, thus making them of interest for practical applications. In Sec. IV the interaction of soliton ratchets with system boundaries is considered, while in Sec. $\mathrm{V}$ we summarize the main result of the paper and discuss possible applications of the phenomenon.

\section{MODEL ANALYSIS}

Direct soliton motion induced by ac signals have been investigated in the literature mainly for ac forces with singleharmonic content [18]. As well known, for symmetric field potentials this situation does not lead to soliton ratchet dynamics. To this regard we remark that the dc motion observed for a sine-Gordon kink driven by single-harmonic forces in absence of damping [19], and its generalization to the case of small damping [20], as well as, dc motion obtained from spatially inhomogeneous drivers [21], should not be confused with soliton ratchets. In these cases, indeed, the $\mathrm{dc}$ motion strongly depends on the initial conditions and quickly disappears as the damping in the system is increased. On the contrary, soliton ratchets do not depend on initial conditions and exist also for relatively higher damping. We remark that net soliton motion independent on initial conditions, can be induced by the mixing of an additive and a parametric (single harmonic) driver as shown in Ref. [22] for solitons of the $\phi^{4}$ model.

In this section we shall investigate soliton ratchetlike dynamics in symmetric potentials driven by periodic biharmonic forces of zero mean. As a working model we take the following perturbed sine-Gordon equation:

$$
u_{t t}-u_{x x}+\sin u=-\alpha u_{t}-E(t)+n(x, t),
$$

with $\alpha$ denoting the damping coefficient, $n(x, t)$ a white noise term with autocorrelation

$$
\left\langle n(x, t) n\left(x^{\prime}, t^{\prime}\right)\right\rangle=D \delta\left(x-x^{\prime}\right) \delta\left(t-t^{\prime}\right),
$$

and $E(t)$ a driver of the form

$$
E(t)=E_{1} \cos \omega t+E_{2} \cos (m \omega t+\theta),
$$

(the case $E_{2} \neq 0$ is referred to as biharmonic driver with even or odd harmonic mixing, depending on $m$ being even or odd). Note that although the symmetry properties of this driver are reduced if $E_{2} \neq 0$ and $\theta \neq 0 \bmod \pi$, the force is periodic, with period $T=2 \pi / \omega$, and has zero mean (the analysis can be generalized to more harmonic components and to arbitrary nonsinusoidal periodic forces). The unperturbed version of Eq. (1) [the perturbation being $\epsilon f(t) \equiv$ $\left.-E(t)-\alpha u_{t}(x, t)\right]$ is the well known sine-Gordon equation with exact soliton (kinks, antikinks) solutions that depend on a free parameter, the velocity $v$ of the kink, which lies in the range $-1<v<1$.

It is of interest to investigate the conditions under which a biharmonic driver of type (3) can induce soliton ratchets in Eq. (1). To this end we remark that due to the translational invariance of the sine-Gordon system (we assume an infinite 
system or a finite one with periodic boundary conditions) we have that to each soliton trajectory with velocity $v$ there is a specular trajectory with the velocity $-v$. One can expect that, in analogy with single particle ratchets [15], only forces which break the $v \rightarrow-v$ symmetry should induce net motion. This argument can be formalized in terms of the kink velocity

$$
v=\frac{1}{2 \pi} \int_{-\infty}^{+\infty} x u_{x t} d x
$$

as follows (note that one could use the momentum of the kink as well, instead of the velocity). Among the possible shifts and reflections in $t, x$, and $u$, we identify the symmetry operations that change the sign of $v$ keeping the sign of the topological charge

$$
Q=\frac{1}{2 \pi} \int_{-\infty}^{+\infty} u_{x} d x,
$$

unchanged (this means that we avoid kink antikink transformations). It is easy to check that there is only one symmetry transformation that changes the sign of kink velocity and leaves the equation of motion unchanged, i.e.,

$$
x \rightarrow-x+x_{0}, \quad t \rightarrow t+\frac{T}{2}, \quad u \rightarrow-u+2 \pi .
$$

This holds true for driving fields satisfying the following condition:

$$
E(t+T / 2)=-E(t) .
$$

We remark that the above symmetry argument accounts only for the breakage of the $v \rightarrow-v$ point-particle symmetry of the soliton, ignoring possible contributions coming from the soliton-phonon interaction [23]. From Eq. (3) we see that condition (7) is always satisfied for drivers with odd mixing while it is always broken for drivers with $m$ even (obviously we take $E_{2} \neq 0$ ). Thus, the $v \rightarrow-v$ symmetry predicts that a sine-Gordon soliton should exhibit a ratchet dynamics when driven by a $m=2$ force [one needs to break the symmetry (7) to get the drift motion], but not when driven by a $m=3$ force.

To the same conclusion one can arrive also from first order perturbation theory, taking as collective coordinate ansatz for the kink profile

$$
u(x, t)=4 \arctan \left(\exp \left[\frac{x-X(t)}{\sqrt{1-\dot{X}^{2}(t)}}\right]\right) .
$$

An ODE for kink's center of mass $X(t)$, can be readily obtained by differentiating the momentum (see Refs. $[19,24]$ ),

$$
P=-\int_{-\infty}^{+\infty} \nu_{x} \nu_{t} d x
$$

with respect to time, and using Eq. (1) and ansatz (8) to simplify the expression. This leads to

$$
\frac{d P}{d t}=-\alpha P+2 \pi E(t)
$$

or equivalently

$$
\dot{v}=-\frac{1}{4}\left(1-v^{2}\right)\left[-\pi \sqrt{1-v^{2}} E(t)+4 \alpha v\right],
$$

where $v(t)=\dot{X}(t)$ and we assumed the usual relativistic relation $P(t)=8 v(t) / \sqrt{1-v(t)^{2}}$ between velocity and momentum to be valid for all times. Equation (10) can be readily solved for $P(t)$, from which the kink velocity can be obtained as

$$
v=\frac{P(t)}{8 \sqrt{1+\frac{P^{2}(t)}{64}}} .
$$

From this equation an analytical expression of the average kink velocity

$$
\langle v\rangle=\frac{\omega}{2 \pi} \int_{0}^{T} v\left(t^{\prime}\right) d t^{\prime}, \quad T=\frac{2 \pi}{\omega},
$$

valid in the limit $E_{j} / \sqrt{\alpha^{2}+(j \omega)^{2}} \ll 1, j=1,2$ (i.e., small momentums or small drift velocities), can be obtained for the case of $m=2$, by expanding the square root in Eq. (12) in series, thus giving

$$
\begin{gathered}
\langle v\rangle=\frac{3}{512} \frac{E_{1}^{2} E_{2} \pi^{3}}{\left(\alpha^{2}+\omega^{2}\right) \sqrt{\alpha^{2}+4 \omega^{2}}} \sin \left(\theta-\theta_{0}\right), \\
\theta_{0}=\arctan \left[\frac{\alpha\left(\alpha^{2}+3 \omega^{2}\right)}{2 \omega^{3}}\right] .
\end{gathered}
$$

From this expression we see that the dependence of $\langle v\rangle$ on the relative phase is perfectly sinusoidal. Similar calculations for the $m=3$ case show that the average kink velocity is zero independently on the value of the relative phase $\theta$ (as well as of an arbitrary initial phase).

These results can be easily understood from the effects of the symmetry properties of the force on the dynamics. In Fig. 1 the force $E(t)$, viewed as a sequence of alternating pulses of equal intensities (i.e., with the same area under the curve) and indicated by dark and light fillings in the figure, is reported for the cases $m=2,3$. We see that for $m=3$ these pulses perfectly balance so that no net motion can arise, while for $m=2$ there is not such a balance (in both cases, however, the average of the force is zero).

In Fig. 2 we report the dynamics obtained from numerical integrations of Eq. (11) for the case $m=2$. We also show the response of the system to the single dark and light pulses composing the force, from which we see that although these pulses have equal intensities, the answer of the system is quite different in the two cases. Note that the negative pulse is more effective than the positive one to produce motion as one can see from the fact that the area under the negative 


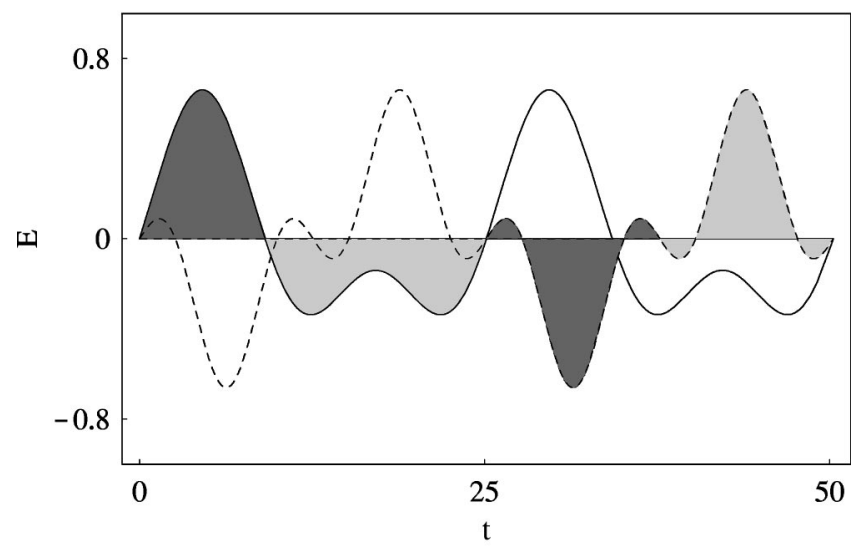

FIG. 1. Profiles of the biharmonic driver for $m=2$ (continuous line) and $m=3$ (dashed line) for parameter values $E_{1}=0.4, E_{2}$ $=0.26, \omega=0.25, \theta=0$. For a better comparison, a time shift of 4.548 and of $-2 \pi$ was, respectively, applied to $m=2$ and $m=3$ cases.

trajectory is greater than the one generated by the positive pulse. This is obviously a consequence of the nonlinearity of the system (in a linear system the area under the two curves would just be the same). For the particular example of Fig. 2 (i.e., $\theta=0$ ) one then expects that a net motion in the negative direction will exist. This is indeed what one finds from integrations of the perturbation Eq. (11) with $\theta=0$ [see Fig. (4) below].

In Fig. 3 the motion of the kink center of mass, $X$, obtained from numerical simulations of Eq. (11) for the cases $m=2,3$, is also reported. We see that while a well defined drift velocity in the $m=2$ case arises, no dc motion is present in the $m=3$ case, this being in agreement with our symmetry analysis.

We also find that the average velocity in Eq. (13), computed after the system reached the steady state regime, depends on the relative phase $\theta$ with a low value that is well approximated by $\langle v\rangle=A \sin \left(\theta-\theta_{0}\right)$, with $A=0.058$ and $\theta_{0}$

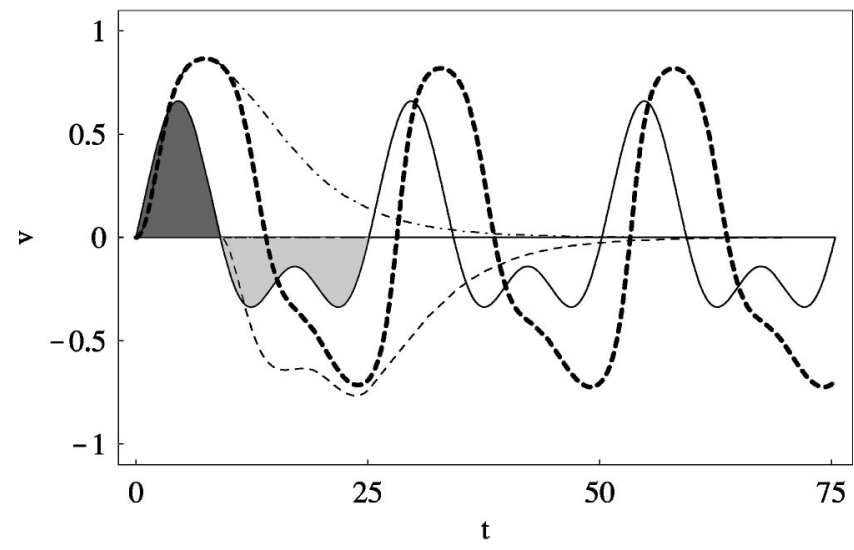

FIG. 2. Time dependence of the velocity $v(t)$ of the kink center of mass (thick dashed curve). The continuous line denotes the force profile, while the dot-dashed and dashed curves represent the response to the single dark and light pulses, respectively. The parameters are the same as in Fig. 1 with $m=2$ and $\alpha=0.15$.

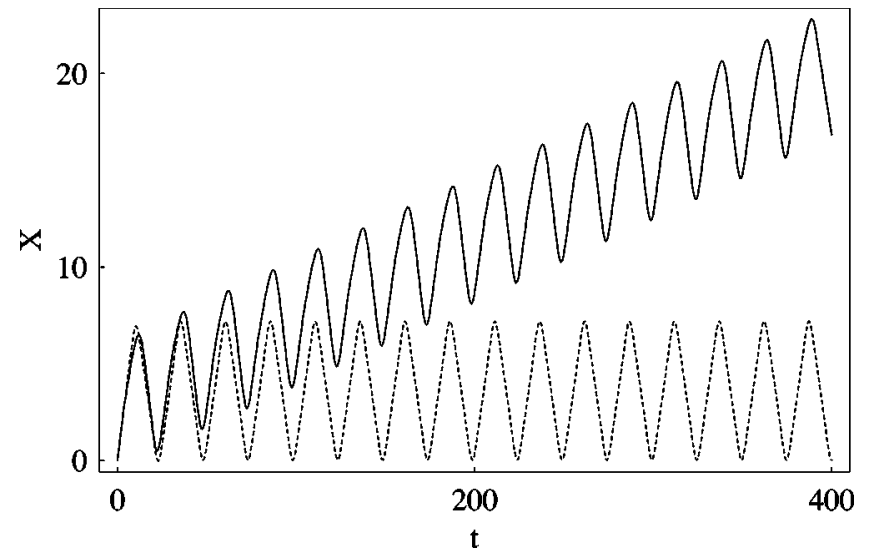

FIG. 3. Trajectories of the kink center of mass derived from Eq. (11) for the cases $m=2$ (continuous curve) and $m=3$ (dashed curve). The other parameters are: $\omega=0.25, \alpha=0.1, E_{1}=0.4, E_{2}$ $=0.26$, and $\theta=1.61$.

$=0.8$. This is shown in Fig. 4 where the dependence of the average velocity on $\theta$, as computed from Eq. (11), is reported with dots, while the continuous line represents the above approximating function. Note that, although the sinusoidal dependence is in perfect agreement with the approximate result in Eq. (14), the explicit values of $A, \theta_{0}$ differ from those predicted by Eq. (14), these being, respectively, $A=0.12, \theta_{0}=0.56$ (this is due to the fact that, for the chosen set of parameters, the approximation of small drift velocities is not valid).

The ratchetlike soliton dynamics discussed above shows similarities with drift phenomena observed in other pointparticle systems. In particular, we mention the unidirectional motion observed for a free particle moving in a nonNewtonian liquid with nonlinear damping under the influence of an additive biharmonic drive [25], and the nonzero drag velocity observed for a point-particle moving on a sinusoidal washboard potential in the overdamped regime, in the presence of the same ac drive with harmonic mixing [26]. In this last case, a dependence of the particle speed on the phase angle of the same type as in Eq. (14) with a phase shift $\theta_{0}$

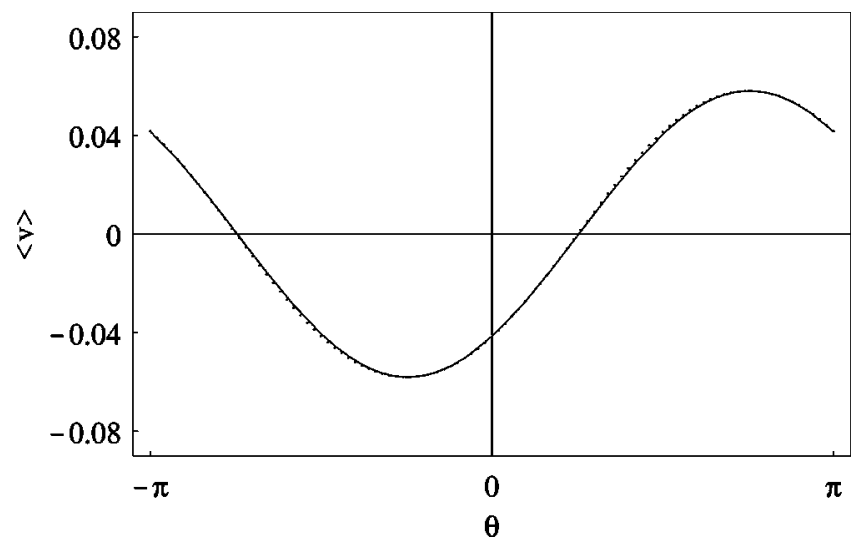

FIG. 4. Average velocity $\langle v\rangle$ of the kink center of mass vs the relative phase $\theta$ for the same parameters as in Fig. 3. The continuous curve refers to the approximating function $\langle v\rangle$ $=0.058 \sin (\theta-0.8)$. 
$=\pi / 2$, was predicted. We remark that the crossover from a sine to cosine dependence of the velocity on the phase angle, occurring at high damping, can be understood in terms of the occurrence of a different symmetry condition [rather than Eq. (7)] that the force should violate in order to provide net motion (see Refs. [4,27] for more details). This is also found in agreement with Eqs. (14), which show that in the limiting cases $\theta_{0} \rightarrow 0$ as $\alpha \rightarrow 0$ and $\theta_{0} \rightarrow \pi / 2$ as $\alpha \rightarrow \infty$ [28].

We also note that from Eq. (14) the point-particle contribution to the drift velocity is expected to be cubic in the driver amplitudes, thus implying that there could be equally important contributions to the soliton velocity at higher orders of the perturbation expansion. In particular, the interaction of the soliton with the phonons in the system, first appearing at second order [17], should not be overlooked. Although the development of a theory that includes second order effects is quite challenging, it is out of the purposes of the present paper (work in this direction is in progress). In the following section will shall instead resort to numerical simulations of Eq. (1) for a full investigation of the problem and for a comparison with the results of the present section.

\section{NUMERICAL STUDIES}

To numerically investigate sine-Gordon soliton ratchets driven by biharmonic fields, we have used standard finite difference schemes to reduce Eq. (1) into a set of ODE that were then integrated in time with a fourth order Runge-Kutta method. To understand the basic mechanism underlying the phenomenon we shall first concentrate on the deterministic case by putting $n(x, t)=0$ in Eq. (1), and then show that the results obtained in this case will survive in the presence of noise.

In Fig. 5 the dynamics of a sine-Gordon kink, initially at rest, driven by a biharmonic driver with $m=2$ and by a single-harmonic driver (i.e., $E_{2}=0$ ), are reported in Figs. 5(a) and 5(b), respectively (note that we use contour plots to show the time evolution surface generated by the kink profile).

From these figures we see that, in the case $m=2$ the soliton center of mass move with a constant drift velocity (note that the shape of the kink during the motion is highly distorted), while for the single-harmonic driver, it oscillates around the initial position (periodic boundary conditions are used in the simulation). This demonstrates the importance of biharmonic drivers in establishing soliton ratchets.

In Fig. 6 we report the dynamics of an antikink ratchet, for the same parameters values as in Fig. 5. We see that, in analogy with soliton ratchets in spatially asymmetric potentials $[9,10,13]$, antikink ratchets move opposite to kinks, the absolute value of the drift velocity being the same.

To check the point-particle perturbation analysis of the preceding section, we have studied the dependence of kink's average velocity on system parameters for the case of a biharmonic driver with $m=2$.

In Fig. 7 we report $\langle v\rangle$ as a function of $\alpha$ for different values of system parameters. Note that the curves display similar behaviors, with a resonance peak in the underdamped regime and a quick decay to zero at higher damping. This
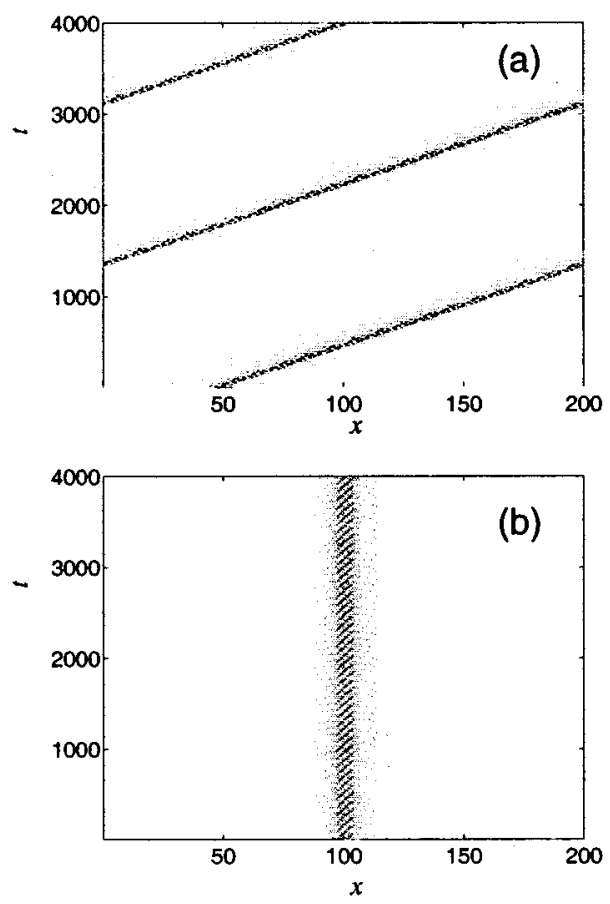

FIG. 5. Contour plots of the velocity field $u_{x}(x, t)$ with $\alpha$ $=0.12, \omega=0.25, \theta=1.61$, in the cases (a) $E_{1}=0.4, E_{2}=0.26$ and (b) $E_{1}=0.66, E_{2}=0$.

behavior is similar to the one reported in Ref. [13] for soliton ratchets in asymmetric potentials, and suggests a possible common mechanism of the phenomenon (see below). We also remark that the interruptions of the curves (cutoffs) at small $\alpha$ values are due to the disappearance of the kink as a consequence of the onset of spatiotemporal chaos in the system (the cutoffs delimit the borders of the existence diagram of the kinks).

Panel (a) of Fig. 7 shows the dependence of $\langle v\rangle$ on $\alpha$ for different values of the driving frequency. Note that the velocity is influenced by resonances with the plasma frequency and its harmonics, as one can see from the nonmonotonous

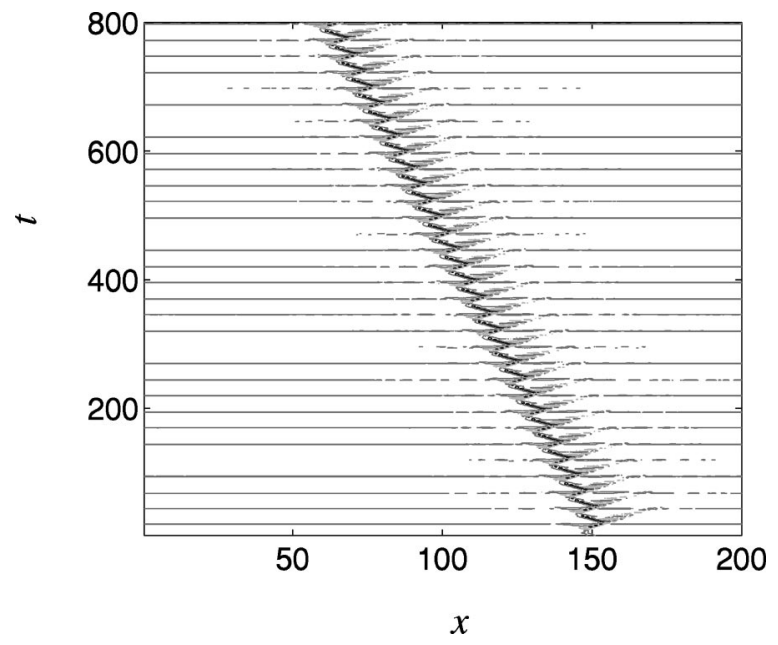

FIG. 6. Contour plot of the velocity field $u_{x}(x, t)$ of the antikink motion. System parameters are as for Fig. 5(a). 

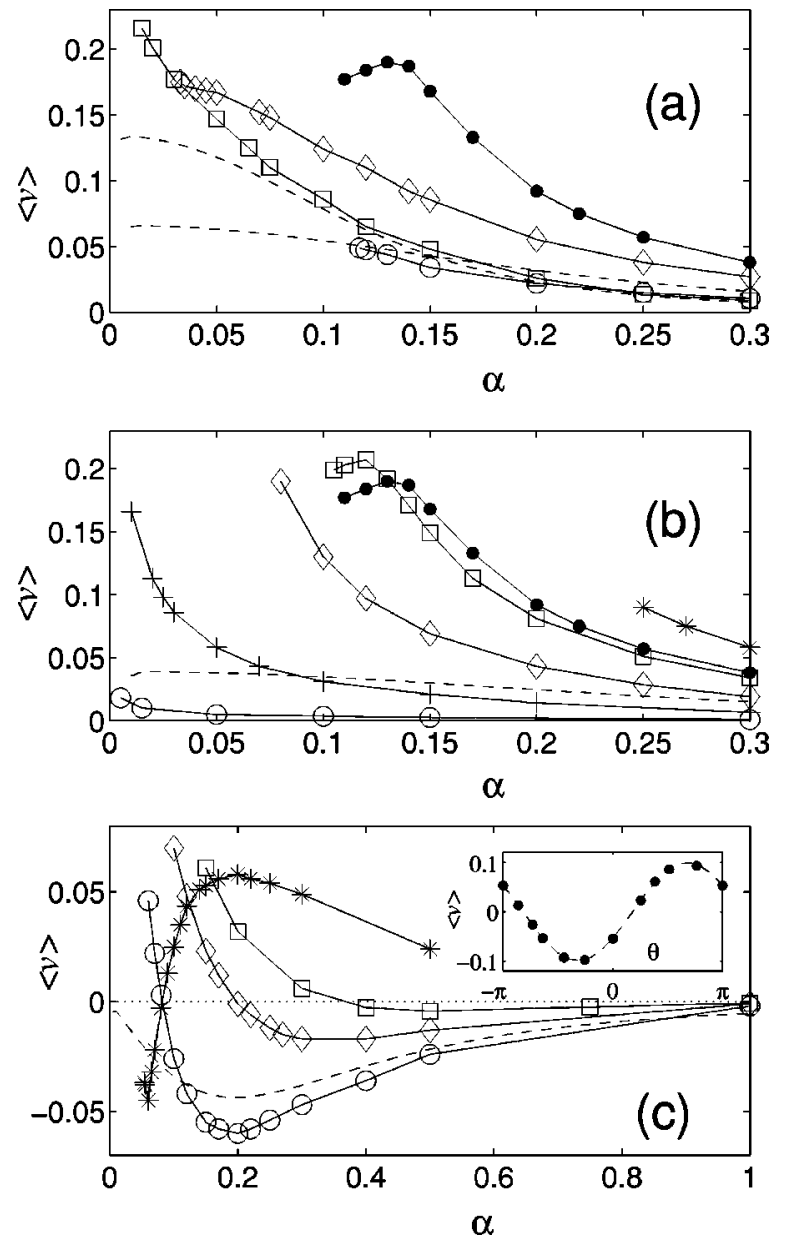

FIG. 7. The averaged kink velocity as a function of damping constant $\alpha$ with $E_{2} / E_{1}=0.65$ for different system parameters. Panel (a): $E_{1}=0.4, \quad \theta=1.61 ; \omega=0.11(\square), \omega=0.25(\diamond), \omega$ $=0.35(\bigcirc)$, and $\omega=0.65(\bigcirc)$. Panel (b): $\omega=0.35, \theta=1.61 ; E_{1}$ $=0.5(*), E_{1}=0.4(\bigcirc), E_{1}=0.38(\square), E_{1}=0.3(\diamond), E_{1}=0.2$ $(+)$, and $E_{1}=0.1(\bigcirc)$. Panel (c): $\omega=0.25, E_{1}=0.4 ; \theta=0(\bigcirc)$, $\theta=0.8(\diamond), \theta=1.2(\square)$, and $\theta=\pi(*)$. The dashed lines show results obtained from the numerical solution of Eq. (11) for $\omega$ $=0.11$ and $\omega=0.25$ in (a), for $E_{1}=0.4$ in (b), and for $\theta=0$ in (c). The inset shows the dependence of the average velocity on the "delay angle" $\theta$ for $\alpha=0.15$ and the rest of parameters as in Fig. 7(c). The dashed curve in the inset shows the fitting curve (see text for details).

behavior of the cutoffs at small $\alpha$ (the cutoff velocities in this case have their largest values close to $\omega=0.5$ and $\omega$ $=1$ ).

Another interesting property emerging from this figure is that the kink velocity is enhanced at low frequencies, and the average velocity decreases by increasing the frequency (already for frequencies above $\omega=1$ directed motion is hardly visible). This is a consequence of the kink inertia to react to fast oscillations. On the contrary drift velocities are observable also at quite small values of $\omega$ (at these values, however, the dynamics becomes complicated and requires long computational times-we checked explicitly the case $\omega$ $=0.01$ for which an average drift is still visible). The dashed lines in the figure represent the results of the point-particle perturbation analysis of the preceding section. We see that the agreement, although qualitatively reasonable, is not so good from a quantitative point of view.

In panel (b) of Fig. 7 the dependence of $\langle v\rangle$ on $\alpha$ is reported for different driver amplitudes (for simplicity we have varied $E_{1}$, fixing the ratio $\left.E_{2} / E_{1}=0.65\right)$. We observe a situation similar to the one shown in Fig. 7(a). By increasing the driver amplitude the system reaches the chaotic regime and cutoff values in $\alpha$ quickly appear. The resonant peak in this case is quite weak and visible only for some narrow range of driving amplitudes. Also here the predictions of the point-particle perturbation theory are quantitatively quite poor. In the panel (c) of Fig. 7 we have shown the dependence $\langle v\rangle(\alpha)$ for different values of the relative phase $\theta$. We see that by changing $\theta$ one can change a maximum of the curve at a given value of $\alpha$, into a minimum. This is a consequence of the sinusoidal dependence of the average velocity on $\theta$ predicted by Eq. (14). To show this, we have reported in the inset of the figure $\langle v\rangle$ vs $\theta$ for a fixed value of $\alpha$, as computed from direct numerical integrations of the sine-Gordon system. We see that the numerical points are well fitted by a sinusoidal law as expected from the first order perturbation result of the preceding section. We remark that a similar sinusoidal dependence was also found in Refs. $[4,26]$ for single ODE particle system, this confirming the existence of a point-particle contribution to the effect. We also note that the dependence of the soliton velocity on $\alpha$ investigated in Fig. 7, displays a $1 / \alpha^{3}$ dependence in the intermediate region $\alpha<1$ (but $\alpha$ not too small) and deviates from it at high value of $\alpha$ where the decay seems to be more exponential-like [28] (in the overdamped limit drift velocities are difficult to measure since due to their smallness they require long computational times).

In experimental situations in which the relative phase between the two drivers is not accessible, one should consider $\theta$ as a random variable, and a final average on it should be taken. The above results then imply that no drift velocity can exist in these cases (soliton ratchets can be induced only if the relative phase $\theta$ remains constant in time).

It is also interesting to note from Fig. 7(c) that reversal currents can be induced by changing the relative phase. In Fig. 8 we show how the curve $\theta=\pi$ of Fig. 7(c), (which displays current reversal at low damping), changes as the driving amplitude is increased. We see that by increasing the amplitude of the driver the kink velocity is increased, this leading to an upwards shift of the curve. This means that current reversal observed for some value of $\theta$ can be removed by properly adjusting the driving amplitude (and vice versa). Note that a further increase of the amplitude can change the shape of the curve destroying the resonantlike character.

In Fig. 9 we report the dependence of the average velocity on the driver amplitude $E_{1}$ with the ratio $E_{2} / E_{1}$ fixed to 0.65 , and with the relative phase between the two drivers $\theta$ $=1.61$. From this figure it is clear that the kink drift velocity depends nonlinearly on the driver amplitude $E_{1}$, with an almost cubic low as one can see from the log-log plot in the inset. This result is in good qualitative agreement with our perturbation analysis (note that the $E_{1}^{2} E_{2}$ dependence in Eq. 


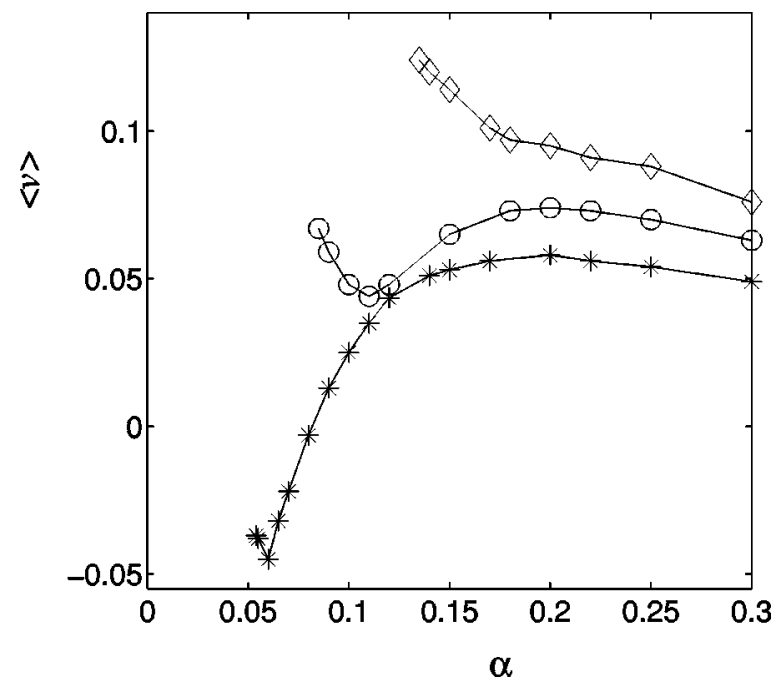

FIG. 8. Dependence of the averaged velocity $\langle v\rangle$ on damping for parameters as in Fig. 7(c) but with different amplitudes: $E_{1}$ $=0.4(*), E_{1}=0.45(\bigcirc)$, and $E_{1}=0.5(\diamond)$. The ratio $E_{2} / E_{1}$ has been kept constant, $E_{2} / E_{1}=0.65$.

(14) implies a $E_{1}^{3}$ dependence if one fixes the ratio $E_{1} / E_{2}$, as done in the numerical simulations). We remark, however, that the case $\omega=0.11$, denoted by $*$ in Fig. 9 , indicates a deviation from this law at higher values of $E_{1}$.

We also checked the predictions of point-particle symmetry arguments and perturbation theory for the case of biharmonic forces with odd harmonic mixing. In Fig. 10 the dynamics of a kink driven by a biharmonic force with $m=3$ is reported. In contrast with the prediction of the preceding section, we see that kink can acquire a drift velocity also in this case. The direction of the motion, however, depends not only on the relative phase, but also on the initial phase (or initial time) of the driving force. By changing the initial

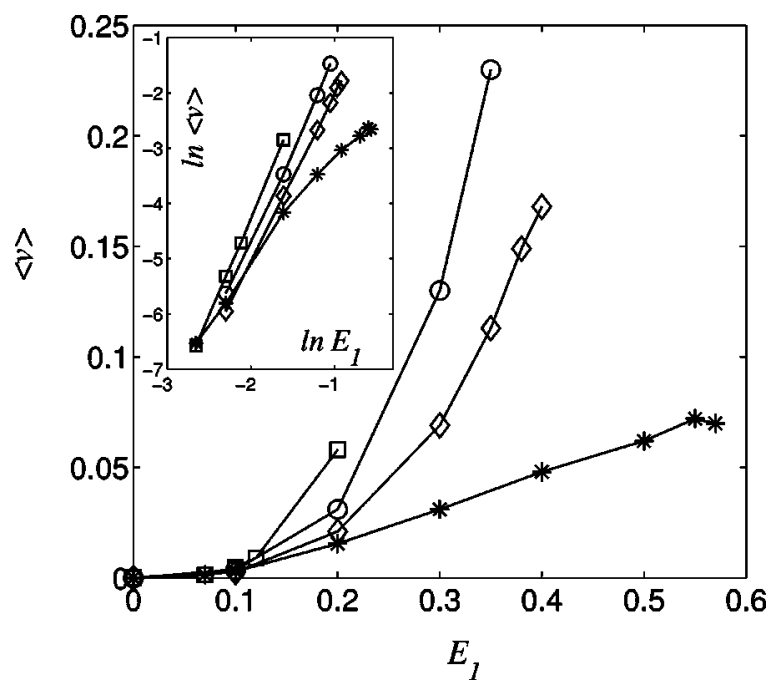

FIG. 9. The averaged kink velocity as a function of the driver amplitude $E_{1}$ with $E_{2} / E_{1}=0.65, \theta=1.61$. The rest of the parameters were fixed as $\omega=0.11, \alpha=0.15(*) ; \omega=0.35, \alpha=0.15(\diamond)$; $\omega=0.35, \alpha=0.1(\bigcirc) ; \omega=0.35, \alpha=0.05(\square)$. The inset shows the log-log dependence.

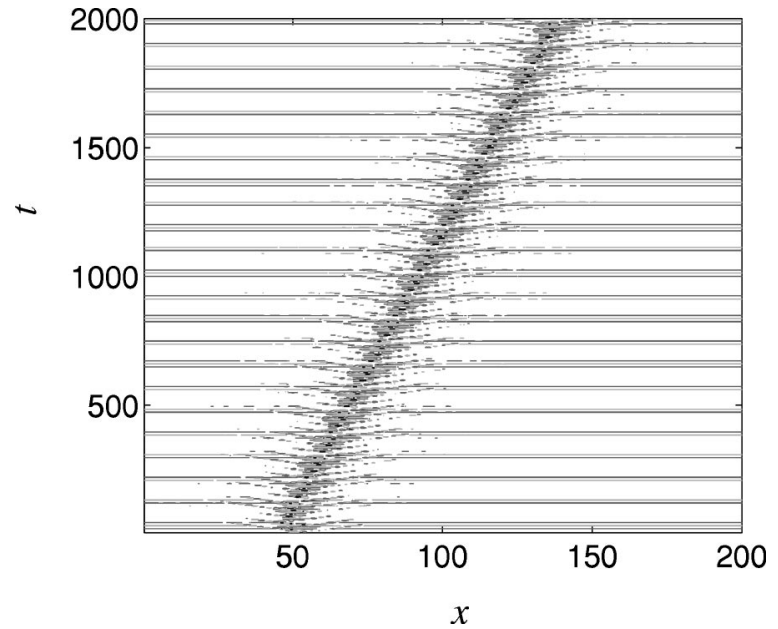

FIG. 10. Contour plot of the velocity field $u_{x}(x, t)$ for a sineGordon kink driven by a biharmonic force with $m=3$. System parameters are as for Fig. 5(a) except $\alpha=0.15$ and $\theta=\pi$.

phase we can achieve soliton moving in the opposite direction with the same velocity, thus by averaging on the initial phase one gets a zero mean velocity for the kink (simulations of the kink dynamics with different initial phases in the interval $[0, T]$ show that roughly half of that interval of initial points yield attraction to kink solutions moving to the right, the other half leading to kink solutions moving to the left with the same velocity). A similar study for the case of $m$ $=2$ did not show any dependence on the initial phase or on initial time. Due to the sensitivity on initial conditions, we conclude that the kink motion in the case $m=3$ is not related to the ratchet phenomenon (this is similar to the cases reported in Refs. [19,20]).

The net motion observed for the $m=3$ case for fixed values of the initial phase, however, is an interesting phenomenon to explore by itself, since it is not linked with pointparticle features of the soliton dynamics (these are excluded by the results of the first order perturbation theory), and is entirely related to the soliton-phonon interaction in a similar way as discussed in Ref. [13].

From the above analysis the following conclusions can be drawn. Although first order perturbation theory captures some qualitative feature of soliton ratchets, it does not provide a satisfactory description of the phenomenon. This is clear both from the fact that there is a poor quantitative agreement between the PDE results and first order perturbation analysis in the case of $m=2$, and from the fact that for $m=3$ it fails to predict the existence of a drift velocity depending on an initial phase.

The reason for this discrepancy is that this analysis includes only point-particle aspects of the soliton dynamics, ignoring completely the internal structure of the soliton. In analogy with the mechanism described in Ref. [13], one could expect a strong contribution to transport coming from the soliton-phonon interaction. Since this interaction arises only at second order in a perturbation expansion, this explains why first order calculations fail to capture the phenomenon.

To elucidate the mechanism underlying soliton ratchets it 

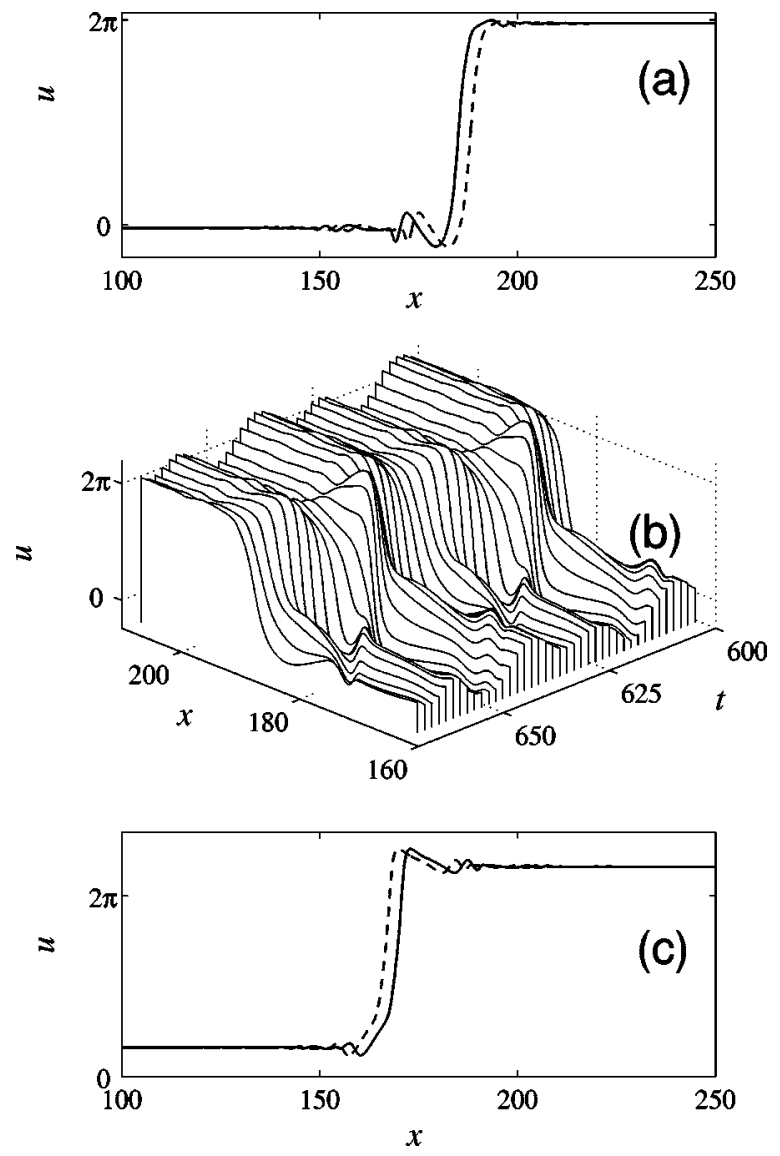

FIG. 11. Kink profile and dynamics for $\alpha=0.15, \omega=0.35, E_{1}$ $=0.5, E_{2}=0.325, \theta=1.61(\mathrm{a}, \mathrm{b})$, and $\theta=1.61-\pi=-1.53$ (c). The profile has been computed at the time moment $t=600$. The dashed line shows the profile of the kink after one period of the external drive.

is useful to investigate the kink dc motion in more details. In Fig. 11 we depict the kink profile while executing the ratchet dynamics in the case of a $m=2$ driver. From this figure the existence of an internal oscillation (internal mode) of the kink profile is clearly seen. Note that the internal mode is strongly desymmetrized with respect to the center of the kink. In the panel (a) the kink moves from the left to the right and the mode appears behind the kink. We checked that the motion of the kink is locked to the external drive. This can be seen from Fig. 11(b), which shows the kink dynamics during two periods of the driving force $E(t)$. This is in agreement with the results of Ref. [13]. The last panel, (c), shows the profile of the kink while moving in the opposite direction than the one in panel (a). Note that when the kink moves to the left, the internal mode is on the right side from the kink center, so it is again behind the kink. This result indicates that there is an evident contribution to the directed kink motion hidden in the asymmetric character of the internal mode and in its interaction with the kink center of mass. The internal mode mechanism can be expected to be effective only in the intermediate damping regime [say $\alpha$ $\approx O(1)]$ since for high damping values oscillations on the kink profile are heavily depressed (the soliton becomes like a rigid particle), while for very low damping internal oscilla-

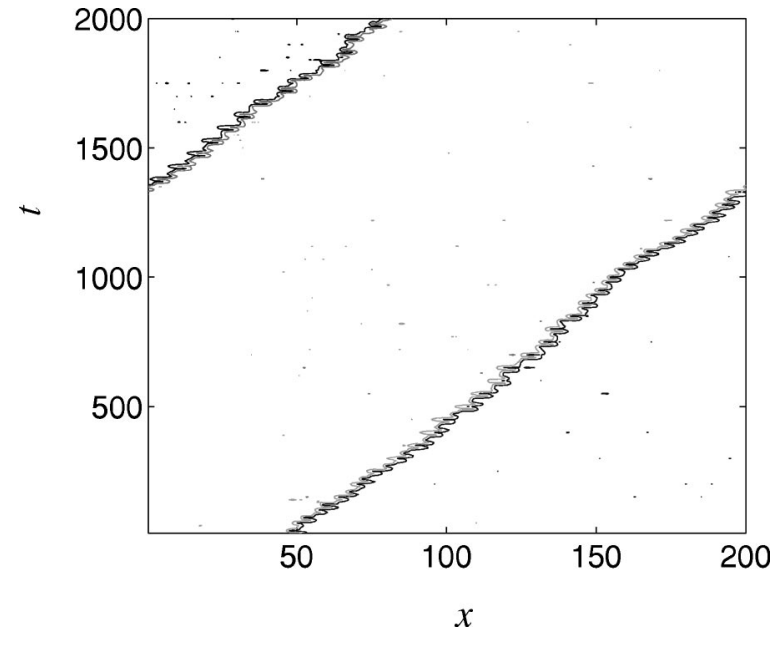

FIG. 12. Countour plot for the displacement field $u(x, t)$ for parameters as in Fig. 1(a) and noise amplitude $D=0.1$.

tions go in competition with incoherent phonon excitations present in the system. We have numerically checked that by increasing the damping the internal mode oscillation become smaller and smaller and almost disappears in the overdamped limit. This correlates with the above numerical results showing a maximum for the drift velocity of the kink at intermediate values of the damping and a rapid decreasing as $\alpha$ is increased.

It is worth to note that although there are no internal modes frequencies in the spectrum of the small oscillation problem around exact soliton solutions of the pure sineGordon equation, these can arise from the perturbation field $\epsilon f$ when it is switched on. This makes the proposed internal mode mechanism for soliton ratchets quite general.

Let us now briefly investigate the influence of a white noise on the kink ratchet dynamics. In Fig. 12 we report the contour plot for the kink motion in the case of a biharmonic driver with $m=2$. We see that the noise introduces disturbances on the profile but does not destroy the drift motion of the soliton. We checked that this property remains true also if we increase the amplitude of the noise up to the kinkantikink nucleation limit. Moreover, the existence of the phenomena in presence of noise shows the validity of the above mechanism also for the nondeterministic soliton ratchets.

\section{BOUNDARY EFFECTS ON SOLITON RATCHETS}

In this section we discuss the effects of the system boundaries on the ratchet dynamics. We have solved the problem for two types of boundary conditions: free ends $u_{x}(0, t)$ $=u_{x}(L, t)=0$ and periodic boundary conditions $u(0, t)$ $=u(L, t)+2 \pi n, n= \pm 1$, where $L$ is the length of the sample. The behavior of kink and antikink solutions does not differ for both cases except, of course, for the behavior at the boundaries. For free boundaries one can show by perturbation theory that the kink may be destroyed at the boundaries if its incoming kinetic energy is below a certain threshold. This can be easily understood since at the boundary the kink undergoes a large-amplitude oscillation that, in the presence of damping, exposes the kink to larger dissipation (the oscil- 

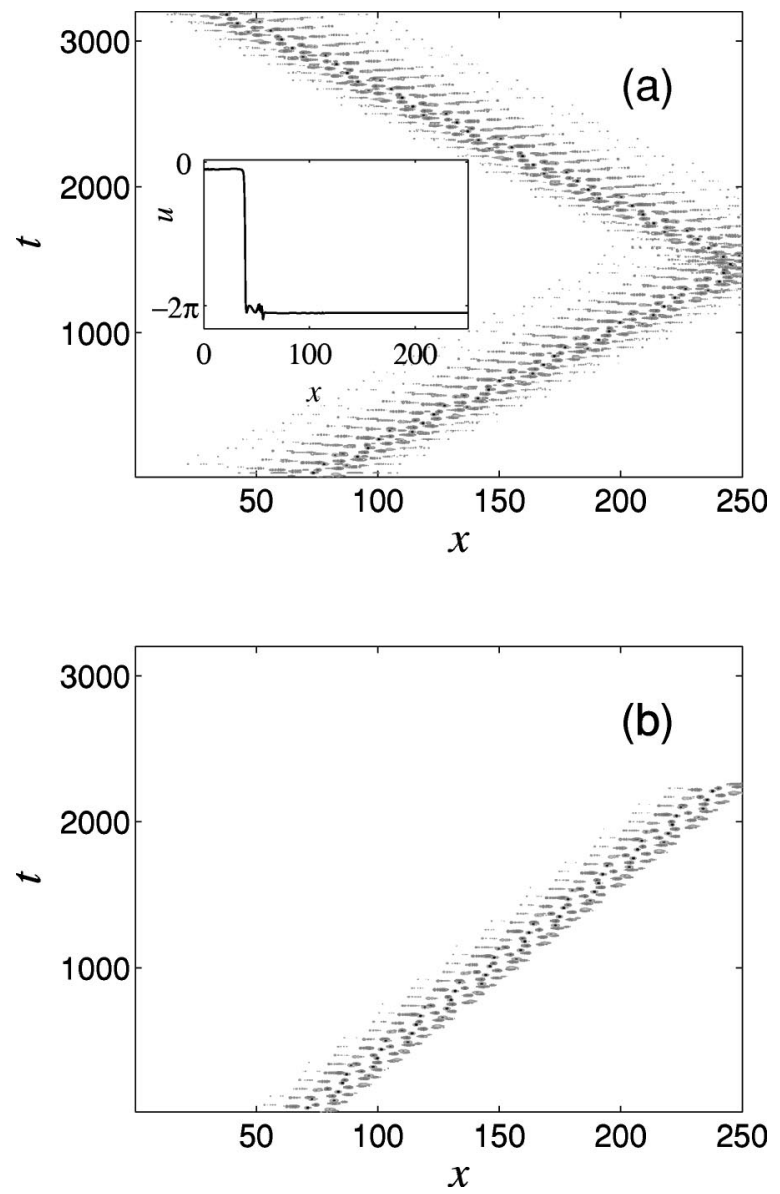

FIG. 13. Contour plots of kink (a) reflection and (b) annihilation at the boundary. The system parameters are $\omega=0.11, E_{1}=0.4, E_{2}$ $=0.26$, and $\theta=1.61$. The damping parameter was $\alpha=0.08$ for the case (a) and $\alpha=0.12$ for the case (b). The inset shows the antikink profile after reflection at time $t=3200$.

lation will be damped and the kink will not be able to attain the $-2 \pi$ value and get reflected as antikink). The synchronization of the soliton motion with the external ac field, as well as the fact that kink and antikink ratchets move in opposite directions, can allow sufficiently energetic soliton ratchets to overcome reflections in the presence of damping. This is clearly shown in Fig. 13(a) where a kink-antikink ratchet reflection is shown.

The possibility to overcome reflection, however, requires overcoming a critical energy threshold that depends on the system parameters. In Fig. 13(b) we show the case in which the soliton ratchet is destroyed at the boundary. At low damping the collision of the kink with the boundary generate oscillations which decay, after some time, leading to the destruction of the kink (increasing the damping the decay time quickly decreases).

The possibility of overcoming reflection depends also on the relative phase of the driver and the internal mode oscillation on top of the kink (this dependence can be tested by shifting the initial incoming positions of the kink). In general, besides kink-antikink reflections and kink destructions, more complicated phenomena can arise. These include the possibility, for particular values of damping, amplitudes, and driver frequencies, to sustain a standing breather at the boundary for very long time. Similar phenomena were also observed in Ref. [29].

To avoid the possibility of ratchet destruction at the boundaries one can recourse to periodic boundary conditions. In this case soliton ratchets, once established, will go on forever (we have checked this numerically for very long computation times). This opens the possibility of interesting physical applications as we will discuss at the end of the next section.

\section{CONCLUSIONS}

In this paper we have considered a way to produce a directed motion of a topological soliton in the presence of damping, by using suitable ac drivers of zero mean. In particular, we showed the possibility of establishing a ratchet dynamics for a kink of the damped sine-Gordon equation when a periodic force, consisting of two harmonic drivers, is applied. In contrast to previous works, the observed dc motion does not require any asymmetry in the potential of system, thus making the phenomena easily accessible to experimental situations.

We also showed that a first order perturbation analysis based on collective coordinates does not provide a complete description of the phenomenon. The reason for this failure was ascribed to the soliton-phonon interaction which, in a perturbation analysis, appears only at second order. In particular we showed that the soliton-phonon interaction manifests with the excitation of an internal mode on the soliton profile that, in the presence of damping, can interact with its translation mode. This provides a basic mechanism to convert the energy of the ac field into direct motion for the soliton that is valid for a wide class of underdamped or moderately damped nonlinear systems. Numerical simulations of the sine-Gordon equation confirm the validity of the proposed mechanism. We also investigated the influence of boundary conditions on the ratchets dynamics in finite sineGordon systems. For reflective boundaries we showed the possibility for soliton ratchet to overcome reflections in presence of dissipation (for periodic boundary conditions the soliton ratchet, once established, will, obviously, go on forever). Finally, we showed that the phenomenon of soliton ratchet is robust enough to overcome the presence of the noise in the system.

This result opens the possibility of interesting applications in different fields. In the context of Josephson junctions, for example, one can predict the existence of a zero field step (i.e., steps in the current-voltage characteristic related to the resonant fluxon motion in the junction) in the absence of dc bias current and in the presence of only biharmonic fields of zero average. This effect should be best observable in annular Josephson junctions where no boundary problems (kink destruction) exist. We also remark that "fluxon-ratchets" inducing zero field steps in Josephson junctions, have not yet been considered both theoretically and experimentally (we will investigate this problem in more detail in a future paper).

Similar transport phenomena can be predicted also in a 
variety of physical systems such as dislocations in crystals, spins waves in magnetic chains, etc. Adjusting the relative phase of the drivers one could control the direction and the velocity of these excitations, this providing a way to control their dynamics. We hope that the results of this paper will soon stimulate experimental work in these directions.

\section{ACKNOWLEDGMENTS}

It is a pleasure to thank P. L. Christiansen, J.C. Eilbeck, S. Flach, Y.S. Kivshar, A.C. Scott, and M.R. Samuelsen for in- teresting discussions. M.S. wishes to thank the MURST for partial financial support through the PRIN-2000 Initiative, and the Department of Physics of the Technical University of Denmark, for a two month Visiting Professorship during which this work was started. Y.Z. acknowledges the hospitality received at the Physics Department of the University of Salerno, where part of this work was done. Both authors acknowledge partial financial support from the European Union grant LOCNET, Project No. HPRN-CT-1999-00163.
[1] R. Z. Sagdeev, D.A. Usikov, and G.M. Zaslavsky, Nonlinear Physics : From the Pendulum to Turbulence and Chaos (Harwood Academic, Cambridge, MA, 1992).

[2] P. Hänggi and R. Bartussek, in Nonlinear Physics of Complex Systems - Current Status and Future Trends, edited by J. Parisi, S.C. Müller, and W. Zimmermann, Lecture Notes in Physics Vol. 476 (Springer, Berlin, 1996) p. 294; F. Jülicher, A. Ajdari, and J. Prost, Rev. Mod. Phys. 69, 1269 (1997); P. Reimann, Phys Rep. 361, 57265 (2002); e-print cond-mat/0010237.

[3] S. Flach, O. Yevtushenko, and Y. Zolotaryuk, Phys. Rev. Lett. 84, 2358 (2000).

[4] O. Yevtushenko, S. Flach, Y. Zolotaryuk, and A.A. Ovchinnikov, Europhys. Lett. 54, 141 (2001).

[5] S. Denisov and S. Flach, Phys. Rev. E 64, 056236 (2001).

[6] M. Barbi and M. Salerno, Phys. Rev. E 62, 1988 (2000).

[7] M. Barbi and M. Salerno, Phys. Rev. E 63, 066212 (2001).

[8] P. Jung, J.G. Kissner, and P. Hänggi, Phys. Rev. Lett. 76, 3436 (1996).

[9] F. Marchesoni, Phys. Rev. Lett. 77, 2364 (1996).

[10] A.V. Savin, G.P. Tsironis, and A.V. Zolotaryuk, Phys. Lett. A 229, 279 (1997); Phys. Rev. E 56, 2457 (1997).

[11] E. Goldobin, A. Sterck, and D. Koelle, Phys. Rev. E 63, 031111 (2001); G. Carapella, Phys. Rev. B 63, 054515 (2001).

[12] F. Falo, P.J. Martínez, J.J. Mazo, and S. Cilla, Europhys. Lett. 45, 700 (1999); E. Trías, J.J. Mazo, F. Falo, and T.P. Orlando, Phys. Rev. E 61, 2257 (2000).

[13] M. Salerno and N.R. Quintero, Phys. Rev. E 65, R025602 (2002).

[14] G. Costantini and F. Marchesoni, Phys. Rev. Lett. 87, 114102 (2001).

[15] K. Seeger and W. Maurer, Solid State Commun. 27, 603 (1978); I. Goychuk and P. Hänggi, Europhys. Lett. 43, 503 (1998); K.N. Alekseev et al., Phys. Rev. Lett. 80, 2669 (1998);
K.N. Alekseev and F.V. Kusmartsev, e-print cond-mat/0012348, and references therein.

[16] M. Salerno, Phys. Rev. B 44, 2720 (1991); G. Filatrella, G. Rotoli, and M. Salerno, Phys. Lett. A 178, 81 (1993); M. Salerno and M.R. Samuelsen, ibid. 190, 177 (1994).

[17] M. Salerno and A.C. Scott, Phys. Rev. B 26, 2474 (1982).

[18] Y.S. Kivshar and B.A. Malomed, Rev. Mod. Phys. 61, 763 (1989).

[19] O.H. Olsen and M.R. Samuelsen, Phys. Rev. B 28, 210 (1983); N.R. Quintero, A. Sánchez, and F.G. Mertens, Phys. Rev. E 62, R60 (2000).

[20] N.R. Quintero and A. Sánchez, Eur. Phys. J. B 6, 133 (1998).

[21] G. Filatrella, B.A. Malomed, and R.D. Parmentier, Phys. Lett. A 198, 43 (1995); experimental observation has been reported in A.V. Ustinov and B.A. Malomed, Phys. Rev. B 64, R020302 (2001).

[22] A.L. Sukstanskii and K.I. Primak, Phys. Rev. Lett. 75, 3029 (1995); see also the related comment by Y.S. Kivshar et al., ibid. 77, 582 (1996).

[23] Stimulated by the results of this paper, S. Flach et al. (see e-print nlin/0110013) have recently provided a general symmetry analysis for soliton ratchets.

[24] D.W. McLaughlin and A.C. Scott, Phys. Rev. A 18, 1652 (1978).

[25] A.K. Vidybida and A.A. Serikov, Phys. Lett. A 108, 170 (1985).

[26] F. Marchesoni, Phys. Lett. A 119, 170 (1986).

[27] P. Reimann, Phys. Rev. Lett. 86, 4992 (2000).

[28] Note that since Eq. (14) is derived for $\alpha$ not too large, extrapolations of the results to the overdamped regime should be made with caution.

[29] P.S. Lomdhal and M.R. Samuelsen, Phys. Rev. A 34, 664 (1986). 